\title{
Análise da Atividade Eletromiográfica de Superfície de Pontos Gatilhos Miofasciais
}

\section{Surface Electromyography Activity Analysis of the Miofascial Triggers Points}

\author{
Aline Bigongiari ${ }^{(1)}$, Patrícia Martins Franciulli( ${ }^{(1)}$, Flávia de Andrade e Souza ${ }^{(1)}$, \\ Luis Mochizuki(2), Rubens Corrêa Araujo ${ }^{(3)}$
}

\section{RESUMO}

Os pontos gatilhos miofasciais (PGMs) são manifestações comumente encontradas na prática clínica e estão relacionados à alteração de tônus e à síndrome dolorosa miofascial (SDM). Objetivo: Avaliar a capacidade da EMG de superfície e detectar alterações da atividade neuromuscular no PGM, em situações de repouso e de contração isométrica voluntária máxima. Método: Participaram do estudo 56 indivíduos distribuídos em dois grupos: grupo Saudável com 28 indivíduos, que necessariamente não possuíam PGM, e o grupo Dor, constituído de 28 indivíduos que possuíam necessariamente PGM no músculo escolhido. O grupo Dor apresentava indivíduos com PGM latente e ativo, e com e sem fenômenos autonômicos (FA). Resultados: O sinal EMG da porção muscular com PGM mostrou-se significativamente maior quando comparado com a porção muscular sadia do grupo Dor, e do grupo Saudável durante o repouso $(26,56 \pm 44,54,5,39 \pm 6,29$ e $1,56 \pm 0,76$, respectivamente, $\mathrm{p}=0,0001)$. Os indivíduos com PGM ativo obtiveram maior intensidade do sinal EMG do que aqueles que apresentaram PGM latente $(17,85 \pm 30,25$ versus $3,74 \pm 1,52, \mathrm{p}=0,04)$. Além disso, os indivíduos que apresentaram fenômenos autonômicos tiveram maior intensidade do sinal EMG do que aqueles que não os apresentaram $(16,78 \pm 28,44$ versus $3,51 \pm 3,65)$, na condição de repouso. Conclusão: A EMG de superfície é capaz de mensurar a atividade do PGM, principalmente na condição de repouso.

Palavras-chave: eletromiografia, ponto gatilho miofascial, tônus muscular.

\section{INTRODUÇÃO}

O tônus muscular pode ser definido como a resistência encontrada à movimentação externa em um estado de relaxamento voluntário ${ }^{(1,2)}$. Existem quadros clínicos de rotina cujas manifestações estão relacionadas à alteração do tônus

\begin{abstract}
There are examples of common clinical conditions that clinical signals are related to alterations in muscle tone, including myofascial pain syndrome. Objective: to discuss the application of surface EMG to detect effect of miofascial trigger point (MTP) on neuromuscular activity at rest and maximum voluntary contraction of the trapezoid muscle. Methods: Fifty-six subjects participated in the study and were divided into two groups: Healthy group $(n=28)$, with subjects who necessarily did not present MTP, and PAIN group $(n=28)$, with subjects who necessarily had MTP. Results: The EMG signal was significantly higher in the muscle portion containing MTP than in the healthy muscle portion in PAIN group and than Healthy group $(26,56 \pm 44,54,5,39 \pm 6,29$ and $1,56 \pm 0,76$, respectively, $p=0,0001)$ during rest. In addition, a higher intensity of the EMG signal was observed in subjects with active MTP than in latent MTP (17,85 $\pm 30,25$ versus 3,74 $\pm 1,52, p=0,04$. Besides, the subjects who presented autonomic phenomena had higher EMG signal than those who had no autonomic phenomena $(16,78 \pm 28,44$ versus $3,51 \pm 3,65$. Conclusion: The surface EMG is reliable to measure the muscular activity of the MTP, mainly at rest condition.
\end{abstract}

Key-words: Electromyography (EMG), myofascial trigger point (mtp), muscle tone.

muscular, como a síndrome dolorosa miofascial (SDM). Segundo Wolens ${ }^{(3)}$, a SDM é uma disfunção neuromuscular local que apresenta regiões sensíveis em bandas musculares tensas, que geram dor referida em áreas distantes ou adjacentes à região sensível. Wheeler et al. ${ }^{(4)}$ caracterizaram a SDM com a presença de músculos em estado de encur-

Recebido em 5/12/2007. Aprovado, após revisão, em 17/9/2008. Declaramos a inexistência de conflitos de interesse.

1. Professor do Curso de Fisioterapia/Laboratório de Biomecânica da Universidade São Judas Tadeu (USJT).

2. Professor da Escola de Artes, Ciências e Humanidades da Universidade de São Paulo (USP).

3. Fisioterapeuta e doutor pelo curso de Fisioterapia/Laboratório de Biomecânica da USIT.

CEP institucional: 1100 (instituição credenciada no Conep) Aprovação: 14/1 1/2003

Endereço para correspondência: Rubens C. Araújo, Universidade São Judas Tadeu, Rua Taquari, 546, 03166-000, São Paulo, SP, e-mail: prof.raraujo@usjt.br 
tamento ou contratura, com aumento do tônus e rigidez, e que contenham pontos gatilhos miofasciais (PGM). Os PGM representam uma área focal de distonia, que pode ser revelada pela eletromiografia $(\mathrm{EMG})^{(5)}$. Fricton, Auvinen e Dukstra $^{(6)}$ relataram que a SDM caracteriza-se pela existência de PGM, que apresenta foco hiperirritável dentro de uma faixa tensa do tecido muscular e/ou associado à fáscia. Nesse mesmo estudo, foram encontradas evidências de mudanças histológicas e ultraestruturais nos PGM, que indicariam o aumento localizado do metabolismo oxidativo, resultando desde a neurodistrofia funcional até a distrofia muscular.

Quando ativos, os PGMs manifestam na pessoa: dor, sensibilidade aumentada e fenômenos autonômicos (vasoconstrição localizada, transpiração, coriza, lacrimação, salivação, atividade pilomotora, distúrbios proprioceptivos, como vertigens, zumbidos e alterações de percepção), com disfunção associada ${ }^{(7)}$.

Os PGMs podem ser classificados, de acordo com seu grau de irritabilidade, como ativos e latentes. O PGM ativo causa dor constante, chegando a incapacitar o músculo acometido. Um PGM latente está clinicamente em "silêncio" com respeito à dor, mas pode causar restrições de movimentos e fraqueza no músculo afetado. Com repouso adequado e a ausência de fatores que acarretaram o aparecimento do PGM ativo, este pode se reverter espontaneamente para o estado latente. Um PGM latente pode persistir por anos, como após o restabelecimento aparente de alguma lesão, existindo a predisposição a ataques agudos de dor, desde o menor trabalho ou alongamento excessivo até uma lesão muscular reincidente. Mas, os PGM latentes podem ser ativados por uma lesão muscular direta, como queda ou torção, por fadiga por causa de esforço excessivo e/ou repetitivo e por estresse emocional ${ }^{(3,8,11)}$.

A ocorrência do PGM varia entre músculos. O músculo trapézio é um dos mais freqüentemente acometidos por PGM. Seis diferentes PGMs, cada qual com seu padrão de dor referida, podem ser encontrados bilateralmente nas fibras superiores, médias e inferiores do músculo trapézio ${ }^{(8,12,13)}$.

A identificação do PGM é necessária nas condições em que uma pessoa refere dor que se espalha para outras partes do corpo. O diagnóstico do PGM precisa ter confiabilidade, e isso requer exatidão, coerência, estabilidade e consistência no procedimento de exame. Tais requisitos para o diagnóstico são atualmente determinados pela habilidade manual do examinador, o que dificulta a padronização ${ }^{(13)}$. Os procedimentos biomecânicos podem ser usados para avaliar as alterações do nível de atividade muscular e do tônus. Entre os procedimentos biomecânicos para examinar a função muscular, destaca-se a $\mathrm{EMG}^{(2)}$.

Estudos sugerem o uso da EMG como método de identificação da PGM $^{(5)}$. Durette et al. ${ }^{(14)}$ avaliaram os PGM por meio da EMG de agulha, após a localização palpatória prévia, e não encontraram atividade elétrica anormal nos músculos estudados, nem sobre o PGM, nem longe dele, sugerindo a ausência de anormalidade nos PGM. Por outro lado, Hubbard e Berkoff ${ }^{(12)}$, utilizando a mesma técnica EMG no músculo trapézio, mostraram atividade espontânea vinda do eletrodo localizado no PGM, por um período maior que 50 minutos. O contrário ocorreu com o eletrodo que não estava sobre o PGM, onde não foi registrada atividade. Assim, os autores concluíram que a atividade elétrica espontânea encontrada no PGM deve-se à estimulação simpática, causando contração com baixo grau de tensão muscular involuntária. A atividade simpática explicaria os sintomas autonômicos associados aos PGM.

Em virtude da controvérsia encontrada na literatura sobre a relação entre o sinal eletromiográfico e os PGMs e ao atual interesse em busca de conhecimento sobre a SDM, esse estudo teve como objetivo principal avaliar a capacidade da EMG de superfície em detectar diferenças nas situações de repouso e de contração isométrica voluntária máxima (CIVM) do músculo trapézio em indivíduos com PGM, comparado aos saudáveis. Outro objetivo foi verificar a diferença na atividade eletromiográfica do músculo com PGM em relação ao músculo sadio, por causa dos graus de irritabilidade, ou seja, se o PGM é ativo ou latente, e os fenômenos autonômicos (FA), que ele possa apresentar.

A partir dos resultados deste estudo, espera-se verificar as seguintes hipóteses: a) no repouso, as pessoas que têm PGM vão apresentar maior atividade muscular; b) na presença da PGM, a intensidade do sinal da EMG associada à relação repouso e CIVM estaria alterada.

\section{$\overline{\text { MATERIAL E MÉTODO }}$}

\section{AMOSTRA}

Este trabalho foi submetido e aprovado pelo comitê de ética local e seguiu todos os procedimentos de ética para pesquisa pela Universidade São Judas Tadeu (USJT).

Participaram do estudo 56 indivíduos distribuídos em dois grupos: grupo Saudável com 28 indivíduos $(21 \mathrm{mu}-$ lheres e 7 homens, 23,1 $\pm 4,2$ anos, 64,2 $\pm 12,5 \mathrm{~kg}$ ) que necessariamente não possuíam PGM. O grupo Dor também foi constituído de 28 indivíduos (23 mulheres e 5 homens, $39,8 \pm 17,6$ anos, $67 \pm 8,3 \mathrm{~kg}$ ) que possuíam necessaria- 
mente PGM no músculo escolhido e o diagnóstico de SDM. O grupo Dor apresentava indivíduos com PGM latente e ativo, e com e sem FA. Os indivíduos foram classificados com e sem FA, de acordo com avaliação prévia e o aparecimento ou não dos sinais clínicos descritos por Travell e Simons ${ }^{(8)}$.

\section{INSTRUMENTAÇÃO}

Para a análise eletromiográfica foi utilizado sistema de EMG de superfície (Pathway mr-25 - Prometheus Group) com as seguintes especificações: dois canais de eletromiografia, eletrodos ativos, distanciados a $10 \mathrm{~mm}$ um do outro, em uma placa retangular, de dimensão 3,5 por $1,5 \mathrm{~cm}$, com pré-amplificador de ganho de dez vezes, faixa de intensidade de $\mathrm{l}$ a $800 \mu \mathrm{V}$, conversão do sinal original para o valor root mean square (RMS), filtro passa-banda de 20 a 500 $\mathrm{Hz}$, taxa do modo comum de rejeição (CMRR) $>110 \mathrm{~dB}$, impedância do eletrodo ativo de $10 \mathrm{G} \Omega$ e alimentação com bateria padrão de $9 \mathrm{~V}$.

\section{PROCEDIMENTOS}

Na escolha do músculo, preconizou-se fazer as medidas no músculo trapézio superior, por ser músculo freqüentemente acometido $^{(8,12,13)}$. Foi feita assepsia da pele com algodão umedecido em álcool e, então, foi preparada para a colocação dos eletrodos da $\mathrm{EMG}^{(15)}$. Logo após esse procedimento, o eletrodo foi fixado à pele por meio de uma fita adesiva de dupla face. Os eletrodos de $\mathrm{AgCl}$ foram colocados no ponto médio entre o ponto motor e a inserção distal do músculo ${ }^{(16)}$. O eletrodo de referência permaneceu a $15 \mathrm{~mm}$ do eletrodo de captação.

Após a colocação dos eletrodos, foi feita a coleta de sinal de EMG do repouso durante um minuto. Na seqüência, os indivíduos realizaram a contração voluntária contra a resistência manual aplicada pelo examinador, de acordo com as provas de função muscular descritas na literatura ${ }^{(17)}$ por mais um minuto para se determinar o nível da EMG durante a CIVM. Esse procedimento foi feito primeiramente no lado direito, e depois repetido para o lado esquerdo, e foi o mesmo para os integrantes dos grupos Saudável e Dor. Além disso, para o grupo Dor, foram registrados os sinais EMG sobre o PGM e sobre uma porção saudável do ventre muscular (PSV). A localização dos PGM no músculo trapézio foi feita de acordo com os critérios descritos por Travell e Simons ${ }^{(8)}$.

\section{TRATAMENTO E PROCESSAMENTO DOS SINAIS}

O sinal EMG fornecido pelo equipamento passou por um filtro analógico passa-banda de 20 a $500 \mathrm{~Hz}$, e, em seguida, foi transformado analogicamente em valores RMS. A partir do valor RMS do sinal, foi determinado o seu valor médio durante o período de repouso e o período de contração voluntária máxima em janelas de um minuto.

Para a comparação da EMG foram adotados os seguintes procedimentos de normalização:

a) para a análise da condição de repouso, nos dois grupos os sinais foram normalizados pelos valores obtidos na CIVM;

b) na análise da condição de CIVM, realizada somente para o grupo Dor, foi feita a normalização do PGM pelo valor obtido na PSV.

\section{ANÁlise estatísticA}

Para analisar o efeito do fator PGM foi feita a análise de variância (ANOVA) de um fator (níveis: Saudável, Dor PGM e Dor PSV). Também foi feita a comparação entre os grupos Dor PGM e Dor PSV. Caso houvesse indicação de efeito do fator, foi usado o teste post hoc Bonferroni $(\mathrm{p}<0,05)$. Da mesma forma, para o grupo Dor, a ANOVA de um fator foi feita para se verificar a presença de FA (níveis: com $\times$ sem) e a atividade do PGM (níveis: ativo $\times$ latente). Após a ANOVA apontar que houve diferença estatística entre os grupos, ou seja, que houve efeito do fator analisado (PGM), o teste post hoc determinou qual foi essa diferença entre os níveis do mesmo fator.

\section{RESULTADOS}

A Figura 1 apresenta as médias e os desvios-padrão do sinal EMG do músculo trapézio superior para os grupos Saudável, Dor PGM e Dor PSV na condição de repouso, que foram, respectivamente, de $1,56 \pm 0,76,26,56 \pm 44,54$ e 5,39 $\pm 6,29$. Os valores foram normalizados pela CIVM. A ANOVA de um fator indicou diferença estatística no EMG de repouso entre os grupos analisados (Saudável, Dor PGM e Dor PSV) $\left(\mathrm{F}_{(2,53)}=5049, \mathrm{p}<0,0001\right)$. Na condição de repouso, o sinal da EMG foi maior no grupo Dor PGM $(\mathrm{p}<0,0001)$.

A Figura 2 mostra as médias e os desvios-padrão do sinal EMG do músculo trapézio superior no PGM normalizado pelo EMG da porção saudável do ventre muscular para o grupo Dor no repouso e na CIVM, cujos valores foram, respectivamente, de 7,78 $\pm 16,77$ e 1,65 $\pm 1,75$. A ANOVA apontou diferença estatística entre as condições (repouso e CIVM) no sinal EMG $\left(\mathrm{F}_{(1,26)}=529, \mathrm{p}<0,0001\right)$. O teste post hoc indicou sinal da EMG maior no repouso $(\mathrm{p}<0,0001)$. 


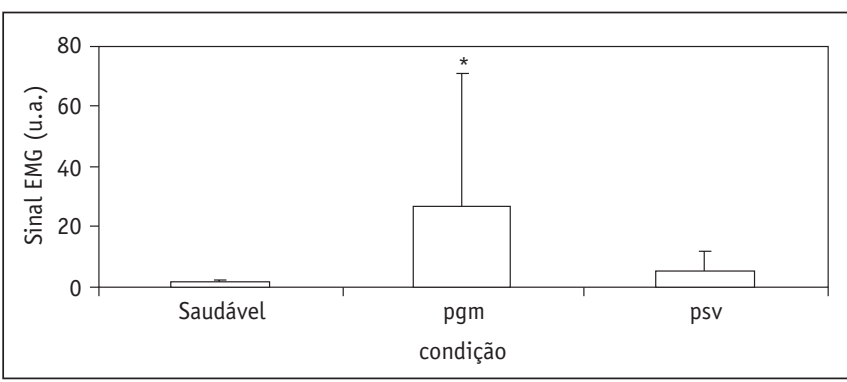

Figura 1 - Análise do sinal EMG do músculo trapézio superior obtido no grupo Saudável e no grupo Dor, com eletrodos colocados sobre o PGM e a PSV, na condição de repouso. Os valores foram normalizados por aqueles obtidos na contração isométrica voluntária máxima. (*) Diferença estatisticamente significante em relação à condição saudável e PSV, $\mathrm{p}<0,05$.

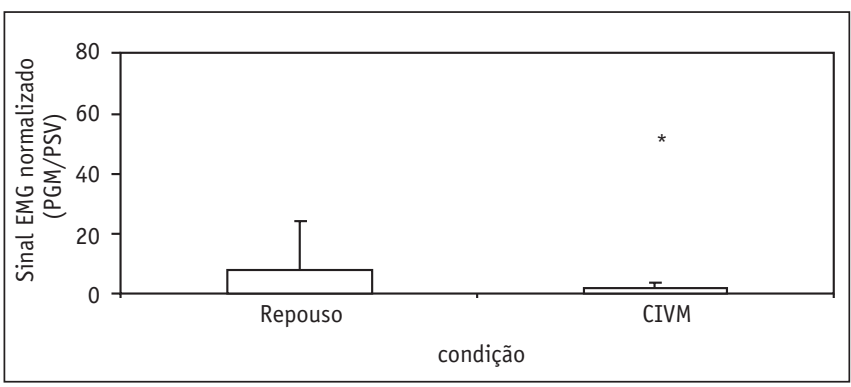

Figura 2 - Análise do sinal EMG do músculo trapézio superior no PGM normalizado pela porção saudável do ventre muscular para o grupo Dor nas condições de repouso e CIVM. $\left({ }^{*}\right)$ Diferença estatisticamente significante, $p<0,05$.

A Tabela 1 apresenta o sinal EMG normalizado pelo sinal obtido na PSV no grupo Dor com pessoas com e sem FA durante o repouso e na CVIM. A ANOVA indicou diferença estatística entre os grupos com e sem FA no sinal EMG $\left(\mathrm{F}_{(1,26)}=4,3 \mathrm{p}=0,04\right)$ durante o repouso. O teste post hoc indicou maior sinal na EMG na presença de FA $(\mathrm{p}<0,05)$. Não houve efeito de FA na condição CVIM sobre a intensidade do EMG.

\section{TABELA 1}

COMPARAC̆̃o DO SINAL EMG NOS INDIVÍDUOS PORTADORES DE PGM COM E SEM FENÔMENOS AUTONÔMICOS (MÉDIA, DESVIO-PADRÃO, AMOSTRA E NÍVEL DE SIGNIFICÂNCIA NAS CONDIÇÕES DE REPOUSO E CONTRAÇÃO VOLUNTÁRIA ISOMÉTRICA MÁXIMA

\begin{tabular}{lccccc}
\hline & Com fenômenos autonômicos & \multicolumn{3}{c}{ Sem fenômenos autonômicos } \\
\hline Condição & & $\mathrm{n}$ & & $\mathrm{n}$ & $\mathrm{p}$ \\
Repouso & $16,78 \pm 28,44$ & 9 & $3,51 \pm 3,65$ & 19 & 0,04 \\
CIVM & $1,28 \pm 0,84$ & 9 & $1,81 \pm 2,05$ & 19 & 0,46 \\
\hline
\end{tabular}

A Tabela 2 apresenta o sinal EMG, normalizado pelo sinal obtido na PSV, entre os portadores de PGM ativos e latentes durante o repouso e na CVIM. A ANOVA de um fator apontou diferença estatística entre os grupos de PGM ativo e latente $\left(\mathrm{F}_{(1,26)}=4,6, \mathrm{p}=0,04\right)$ apenas no repouso. O teste post hoc mostrou maior EMG no PGM ativo $(\mathrm{p}<0,05)$. Não houve efeito de FA na condição CVIM sobre a intensidade do EMG.

\section{TABELA 2}

COMPARAÇ̃̃O DO SINAL EMG NOS INDIVÍDUOS PORTADORES DE PGM ATIVOS E LATENTES (MÉdia, DESVIO-PADRÃO, AMOSTRA E NÍVEL DE SIGNIFICÂNCIA) NAS CONDIÇÕES DE REPOUSO E CONTRAÇÃO VOLUNTÁRIA ISOMÉTRICA MÁXIMA

\begin{tabular}{lccccc}
\hline & PGM ativo & \multicolumn{4}{c}{ PGM latente } \\
\hline Condição & & $\mathbf{n}$ & & $\mathbf{n}$ & $\mathbf{p}$ \\
Repouso & $17,85 \pm 30,25$ & 8 & $3,74 \pm 1,52$ & 20 & 0,04 \\
CIVM & $1,05 \pm 0,63$ & 8 & $1,88 \pm 2$ & 20 & 0,26 \\
\hline
\end{tabular}

\section{DISCUSSÃO}

A primeira hipótese deste estudo se refere à busca pela confirmação de que a atividade muscular seria alterada pela presença dos PGM. Esta hipótese foi confirmada à medida que a atividade do grupo Saudável apresentou menor atividade que o grupo Dor PGM. Este resultado fortalece a utilização da EMG como forma de identificar os PGM ${ }^{(18-20)}$ e no auxílio do diagnóstico de síndromes dolorosas ${ }^{(21)}$.

Simons e Mense ${ }^{(21)}$ acreditam que o tônus muscular depende de dois fatores: propriedades viscoelásticas básicas associadas ao grau de ativação voluntária e/ou involuntária do músculo. Na cefaléia tensional, por exemplo, termo comumente usado para designar dores de cabeça de origem miogênica, em que a presença de PGM é comum, o músculo trapézio tem papel fundamental, apresentando-se, na maioria dos casos, com espasmos (contrações involuntárias), podendo ser reconhecida atividade EMG na situação de repouso ${ }^{(21)}$.

O exame de palpação sobre a região onde o PGM está presente também sugere aumento da rigidez local ${ }^{(7,8,22)}$, e isso foi notado durante a preparação dos indivíduos para o teste deste estudo. Os resultados do presente estudo sugerem que os PGMs estão associados à alteração da atividade muscular, o que pode ser proveniente de disfunção neuromuscular ${ }^{(23,24)}$. Entretanto, a análise de provável disfunção neuromuscular precisa ser investigada futuramente ${ }^{(23-25)}$.

Hubbard, Berkoff ${ }^{12)}$ e Simons ${ }^{(23)}$ propuseram que a razão para essa liberação excessiva de acetilcolina e, portanto, a atividade EMG elevada, deve-se à modulação autonômica 
que, por sua vez, é estimulada pela liberação de substâncias químicas comumente encontradas no tecido muscular alterado, concluindo que, ao modificar o estímulo do sistema nervoso simpático, ocorrem alterações no tônus muscular.

A segunda hipótese deste estudo se fundamenta em uma constatação muito simples: a atividade eletromiográfica no repouso é menor que a atividade durante a CIVM. De fato, esta expectativa é gerada porque no repouso a quantidade de potências de ação sendo disparadas é muito baixa ${ }^{(24)}$. Portanto, em condições de CIVM espera-se aumento considerado de disparos de unidades motoras e conseqüente aumento da intensidade do EMG. Nessa condição, os efeitos neuromusculares do treinamento físico, por exemplo, são observáveis na condição CIVM e não no repouso. Entretanto, o que notou-se no presente estudo foi que na CIVM não registrou diferença entre os grupos com e sem fenômenos autonômicos ou do fato do PGM ser ativo ou latente. As diferenças entre esses dois grupos somente foram identificadas durante o repouso. Logo, a relação da intensidade dos sinais da EMG associada à relação repouso e CIVM é alterada na presença da PGM, confirmando a segunda hipótese deste estudo.

Uma conseqüência metodológica do presente trabalho diz respeito sobre qual condição é mais propícia para estudar a PGM. Para se analisar atividade muscular nos PGM comparado à PSV do mesmo músculo ou comparada a um sujeito normal, a melhor situação é a de repouso e não a de contração muscular. Igualmente importante é a com-

\section{REFERÊNCIAS}

1. Pisano F, Miscio G, Colombo R, Pinelli P: Quantitative evaluation of normal muscle tone. J Neurol Sci 135: 168-72, 1996.

2. Basmajian JV, De Luca CJ: Description and analysis of the EMG signal. Muscle alives: their functions revealed by electromyography. 5a ed. Baltimore: Williams \& Wilkins; 1985.

3. Wolens D: The miofascial pain syndrome: a critical appraisal. Am Phys Med Rehabil 12(2): 299-316, 1998.

4. Wheeler AH, Aaron GW: Muscle pain due to injury. Curr Pain Headache Rep 5: 441-6, 2001.

5. Rivner MH: The neurophysiology of myofascial pain syndrome. Curr Pain Headache Rep 5: 432-40, 2000.

6. Fricton JR, Auvinen MD, Dukstra D: Myofascial pain syndrome: electromyographic changes associated with local twitch response. Arch Phys Med Rehabil 66: 314-7, 1985.

7. Simons DG, Travell JG: Myofascial trigger points, a possible explanation. Pain 10: 106-9, 1981.

8. Travell JG, Simons D: Background and principles. Myofascial pain and dysfunction. the trigger point manual. Baltimore, Williams \& Wilkins; 1983. paração do nível de atividade muscular na região do PGM e na região do PSV em um mesmo indivíduo.

Nesse estudo, destacou-se que os grupos Dor PGM ativos e com FA apresentaram maior atividade muscular que os do grupo Dor PGM latentes e sem FA, respectivamente. Essa diferença sugere relação direta do grau de irritabilidade do PGM com o sinal da EMG.

Embora a EMG de superfície possa ser ferramenta útil para a mensuração quantitativa dos PGMs, existem poucos trabalhos sobre esse assunto. Os resultados do presente estudo estão de acordo com os de Fricton, Auvinen e Dukstra $^{(6)}$, Hubbard e Berkoff ${ }^{(12)}$ e McNulty et al. ${ }^{(26)}$, exceto pelo fato de que esses trabalhos foram realizados com eletrodos de agulha. Assim, abre-se a perspectiva da realização de estudos que permitam padronização confiável da EMG de superfície, em que muitos clínicos poderiam utilizar tal procedimento para a avaliação objetiva dos PGMs e talvez para o monitoramento dos resultados de seus tratamentos. Além disso, a comparação do sinal EMG de superfície sobre o PGM e sobre a PSV pode ser procedimento de biofeedback para o paciente, o que aumenta as opções de tratamento desse problema.

Concluindo-se, este estudo mostra que a EMG de superfície pode auxiliar na identificação dos PGM, bem como determinar o seu grau de irritabilidade. E, finalmente, para interpretação mais segura do sinal da EMG dos PGM, sugere-se compará-lo com a atividade muscular de uma parte saudável do músculo.

9. Jacob A: Miofascial pain. Phys Med Rehab 5(3), 1991.

10. Musse C: Síndrome dolorosa miofascial. In: Lianza, S. Medicina de reabilitação. Rio de Janeiro: Guanabara Koogan; 1995.

11. Ensenyel M, Caglar N, Tayfun A: Treatment of miofascial pain. Am J Phys Med Rehabil 79(1): 48-52, 2000.

12. Hubbard DR, Berkoff GM: Myofascial trigger point show spontaneous needle EMG activity. Spine 18(13): 1803-7, 1993.

13. Gerwin RD, Shannon S, Hong CZ, Hubbard D, Gevirtz R. Interrater reliability in myofascial trigger point examination. Pain 69: 65-73, 1997.

14. Durette MR, Rodriquez AA, Agre JC, Silverman JL: Needle electromyographic evaluation of patients with myofascial or fibromyalgic pain. Am J Phys Med Rehabil 70(3): 154-6, 1991.

15. Snyder-Mackler L, Robinson AJ: Eletrical stimulation of muscle. techniques and applications. clinical electrophysiology electrotherapy and electrophysiologic testing. 2a ed. Baltimore; Williams \& Wilkins; 1995.

16. Hermes HJ, Freriks B, Disselhorst-Klug C, Rau G: Development of recommendations for SEMG sensors and sensor placement procedures. J Electromyogr Kinesiol 10: 361-74, 2000. 
17. Kendall FP, Mc Creary E, Provance PG: Prova de força de membro superior e cintura escapular. músculos: provas e funções. 2a ed. São Paulo: Manole; 1995.

18. Hwang M, Kang YK, Kim DH: Reffered pain pattern of the pronator quadratus muscle. Pain 116 (3): 238-42, 2005.

19. Treaster D, Marras WS, Sheedy JE, Hart D: Myofascial trigger point development from visual and posturasl stressors during computer work. J Electromyogr Kinesiol 16(2): 115-24, 2005.

20. Kuan TS, Hsieh YL, Chen JT, et al.: The miofascial trigger point region: correlation between the degree of irritability and the prevalence of endplate noise. Am J Phys Med Rehabil 86(3): 183-9, 2007.

21. Simons DG, Mense S: Understanding and measurement of muscle tone as related to clinical muscle pain. Pain 75: 1-17, 1998.
22. Niddam DM, Chan RC, Lee SH, et al.: Central representation oh hyperalgesia from myofascial trigger point. Neuroimage 1-8, 2007.

23. Simons DG: Review of enigmatic MTrPs as a commom cause of enigmatic musculoskeletal pain and dysfunction. J Electromyogr Kinesiol 14: 95-107, 2004.

24. Bennett R: Myofascial pain syndromes and their evaluation. Best Pract Res Clin Rheumatol 21(3): 427-45, 2007.

25. Yap EC: Myofascial pain - an overview. Ann Acad Med Singapore 36: 43-8, 2007.

26. Mcnulty WH, Gevirtz RN, Hubbard DR, Berkoff GM: Needle electromyographic evaluation of trigger point response to a psychological stressor. Psychophysiology 31: 313-6, 1994. 